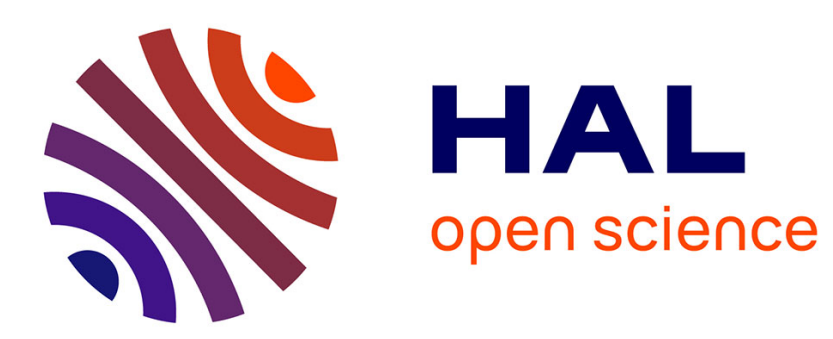

\title{
Dispersion scolaire et politiques publiques contre le décrochage en Italie
}

Thierry Berthet

\section{To cite this version:}

Thierry Berthet. Dispersion scolaire et politiques publiques contre le décrochage en Italie. Formation Emploi. Revue française de sciences sociales, 2019, 147, pp.89-112. 10.4000/formationemploi.7643 . halshs-02426149

\section{HAL Id: halshs-02426149 \\ https://shs.hal.science/halshs-02426149}

Submitted on 12 Feb 2020

HAL is a multi-disciplinary open access archive for the deposit and dissemination of scientific research documents, whether they are published or not. The documents may come from teaching and research institutions in France or abroad, or from public or private research centers.
L'archive ouverte pluridisciplinaire HAL, est destinée au dépôt et à la diffusion de documents scientifiques de niveau recherche, publiés ou non, émanant des établissements d'enseignement et de recherche français ou étrangers, des laboratoires publics ou privés. 


\title{
Dispersion scolaire et politiques publiques contre le décrochage en Italie
}

\author{
Thierry Berthet \\ Directeur de recherche en science politique au CNRS. \\ Directeur du LEST, Laboratoire d'Économie et de Sociologie du Travail, \\ Aix-Marseille Univ, CNRS, LEST, Aix-en-Provence, France
}

\begin{abstract}
Resumé
Cet article propose une analyse des politiques de lutte contre le décrochage scolaire en Italie. Après une présentation du système scolaire italien, la notion communément utilisée de dispersion est discutée, au regard d'une conception fondée sur l'inclusion scolaire. La présentation des chiffres retenus pour dénombrer les décrocheurs met ensuite en relief des différences régionales fortes $\mathrm{La}$ discussion de la continuité des politiques éducatives italienne permet d'engager une seconde partie consacrée aux politiques publiques. Basée sur une enquête de terrain en région Lazio (Latium), elle aborde la mise à l'agenda du décrochage, la structure de la politique publique de lutte contre la dispersion scolaire et les instruments mobilisés par les établissements d'enseignement secondaire.
\end{abstract}

\section{Abstract \\ School dispersion and public policies against early school leaving in Italy}

This article provides an analysis of early school leaving policies in Italy. After briefly setting the context of the Italian school system, the commonly 
used notion of dispersion is presented with regard to a conception based on school inclusion as well as the data used to count dropouts, which reveal strong regional differences. As a follow-up of the discussion regarding the continuity of Italian educational policies, the second part of the article devoted to the study of early school leaving public policies. This section, based on a field research in the Lazio region, deals with the setting of the dropout agenda, the structure of public policy aimed at curbing school dispersion and the various instruments used by secondary schools in an everyday effort to promote school perseverance.

Mots clés : Décrochage scolaire, Italie, action publique, politique régionale, instruments d'action publique.

Keywords: School drop-out, Italy, public action, early school leaving, regional policy, instruments of public action.

Quand un chercheur, politiste et français, travaillant sur le décrochage scolaire, arrive en Italie ${ }^{1}$ pour essayer de comprendre comment sont conçues et mises en œuvre les politiques de lutte contre l'abandon scolaire, il est forcément surpris. Surpris d'abord par les termes employés. Le terme de décrochage est absent du lexique italien et il entend surtout parler de dispersion scolaire, parfois d'abandon. Voire même, à sa grande stupéfaction, il arrive qu'on lui parle de «mortalité scolaire » pour désigner la désertion des bancs de l'école. Surpris également par l'organisation de l'enseignement secondaire laissant une très large autonomie aux établissements. La première marche à gravir pour le chercheur néophyte transalpin consiste donc à se familiariser avec un système scolaire qui lui est étranger. Comprendre l'architecture d'un système scolaire n'est jamais chose facile. La raison en est à la fois simple à comprendre, mais difficile à appréhender au concret. Un système scolaire, c'est avant tout, au moment où on l'étudie, un héritage historique sédimenté dans le temps. Ici, à Rome, il témoigne de la réunification d'un pays longtemps morcelé en principautés territoriales, de la monarchie, puis du fascisme en son sein, enfin, d'une république incertaine, mais qui dure pourtant. Tous ces régimes s'y sont

\footnotetext{
${ }^{1}$ Ce travail de recherche a été réalisé à l'occasion d'un séjour d'étude de sept mois au sein de l'École Française de Rome et je tiens à remercier très chaleureusement la direction de l'école et des études contemporaines pour la qualité de leur accueil.
} 
succédés et tous ont laissé des traces dans les écoles italiennes. L'école est en effet l'un de ces secteurs d'action publique (Jobert \& Muller, 1987) incarnés dans de grandes institutions régaliennes où la dépendance au sentier $^{2}$ (Ghezzi \& Mingione, 2007 ; Palier, 2010) joue à plein régime son rôle de sédimentation. Les réformes se succèdent au fil des ministères et chacune apporte sa couche institutionnelle, chacune laisse sa petite marque. Cet article débute donc par une rapide mise en perspective des points clés du système scolaire italien et des grandes lignes de ce qui, en son sein, vise à apporter des solutions à un problème qui, pour le coup, ne connaît pas de frontières nationales : l'abandon de l'école avant d'en sortir diplômé, ne futce que minimalement. Mais avant d'en venir aux solutions, il faut d'abord savoir de quoi l'on parle. Et en l'occurrence saisir les termes du débat, mettre au clair les chiffres et comment ils sont construits. Savoir ce qu'abandonner l'école veut dire et le lien qui est fait mécaniquement entre absentéisme et décrochage. Il convient également revenir sur la continuité des institutions publiques qui ont en charge la gestion de cette question sociale dès lors qu'elle devient un problème public (Neveu, 2015). Et, faits marquants s'il en est en Italie, revenir sur les disparités régionales qui constituent une variable clé de toute compréhension des problèmes politiques en Italie.

Alors on peut, dans une seconde partie, en venir à l'étude de l'action publique en matière de lutte contre le décrochage. Elle est guidée par un questionnement faisant le lien entre conception du décrochage et nature de l'offre publique d'intervention/remédiation. On s'inscrit ainsi dans une perspective d'approche cognitive des politiques publiques (Muller 2000 ; Sabatier \& Schlager, 2000) néo-institutionnaliste postulant un lien de causalité entre la représentation d'un problème social et les politiques publiques afférentes. Pour le dire autrement, la conception fondée sur une approche globale de l'inclusion scolaire produit-elle une offre publique de dispositifs spécifique ? Cette seconde partie est séquencée en trois temps : la mise sur agenda politique du décrochage scolaire et ses registres de légitimation, l'organisation des politiques publiques italiennes contre la dispersion scolaire et enfin les dispositifs mis en œuvre dans les

2 «La notion de path dependance ou dépendance au chemin empruntée ici s'est développée en science politique au cours des années 1990, pour souligner le poids des choix effectués dans le passé et celui des institutions politiques sur les décisions présentes" (Boussaguet, Jacquot \& Ravinet, 2006, p. 20). 
établissements qui constituent le cœur opérationnel de la lutte contre le décrochage.

\section{Encadré 1. Méthodologie}

Le matériau empirique qui appuie cet article est adossé à une campagne d'entretiens $(n=22)$ menés durant l'année 2016-2017 auprès d'une série d'acteurs nationaux (ministère italien de l'Éducation, organisme de formation et experts du domaine), régionaux, tels que l'USR (Ufficio Scolastico Regionale, équivalent fonctionnel du rectorat) et le Conseil régional de la région Lazio (Latium) et locaux, sur la base d'une dizaine d'établissements de la région Lazio qui a pour cœur la métropole romaine.

Ces derniers entretiens ont été conduits par voie semi-directive auprès des dirigeants et de quelques enseignants directement impliqués dans la stratégie de l'établissement en matière de décrochage. Ils couvrent des situations contrastées mêlant des établissements secondaires supérieurs relevant de plusieurs types de filières: lycée d'enseignement général, instituts techniques ou instituts professionnels. Ils couvrent également des situations géographiques distinctes : établissement renommé de centre-ville, de périphérie métropolitaine, de ville moyenne, de zones de montagne ou de mer.

Construit par effet boule de neige, avec le concours du Consulat de France et l'USR Lazio, cet échantillon - qui ne vise pas la représentativité, mais la diversité des situations - s'est concentré sur les établissements publics.

Les entretiens ont été conduits en italien, puis retranscrits et traduits en français. Ils ont été ensuite exploités autour de huit thèmes: chiffres du décrochage ; représentations $\mathrm{du}$ phénomène; ordre scolaire (articulation formations initiale/continue, générale/professionnelle, etc.) ; chronologie de la mise à l'agenda politique ; Instruments et dispositifs ; dimension territoriale et mobilités ; Expertise et connaissance, et finalement prise en compte des capacités individuelles. L'analyse de cette campagne d'entretiens a été précédée par une large étude documentaire incluant rares écrits scientifiques sur le sujet, textes législatifs, rapports d'étude et parlementaires, documents techniques et au niveau des établissements : documents de présentation et de programmation notamment les Plans triennaux de l'offre de formation (PTOF), les rapports d'autoévaluation (RAV), les plans d'amélioration de l'établissement (PAE), les plans annuels d'inclusion (PAI). Ils sont en général disponibles avec des données sur les établissements par l'intermédiaire du système de monitoring Scuola in chiaro (http://cercalatuascuola.istruzione.it). 


\section{Le contexte scolaire italien et l'abandon d'études : une question de dispersion}

Comme bien des systèmes scolaires, l'école italienne est en constante évolution, en réforme permanente au rythme des alternances gouvernementales. L'objet de cette première partie descriptive est de fournir quelques clés de compréhension de l'architecture du système éducatif italien et de la place qu'y tient la problématique du décrochage scolaire.

Elle est cadencée en cinq temps : une présentation générale du système scolaire italien, de la notion de dispersion scolaire, des chiffres du décrochage scolaire, de la discontinuité des politiques éducatives et, pour finir, des disparités régionales qui marquent le phénomène du décrochage scolaire, comme de nombreux autres problèmes sociaux italiens.

\subsection{Le système scolaire italien : quelques balises}

Le système scolaire italien est structuré autour d'une série de cycles dont l'organisation a été introduite, en 2000 par la réforme Berlinguer. En préambule à la scolarité obligatoire, les crèches et écoles maternelles relèvent principalement de la compétence des communes. L'entrée en scolarité se fait de manière optionnelle à partir de trois ans dans les écoles maternelles (Scuola dell' infanzia) qui sont formellement placées sous la compétence du ministère de l'Instruction publique (Ministero dell'Istruzione, dell'Università e della Ricerca-MIUR) notamment pour la partie pédagogique, mais gérées de fait par les communes voire des organisations privées.

La scolarité obligatoire débute avec l'entrée à l'école primaire (Scuola primaria) à partir de six ans (de six à dix ans). L'école primaire dure cinq années. Le temps scolaire varie selon le choix des familles entre 24 et 40 heures hebdomadaires ${ }^{3}$.

L'enseignement secondaire est divisé en deux cycles: le secondaire de premier degré, équivalent au collège français (Scuola media), et le secondaire de second degré ou supérieur correspondant au lycée français. Le secondaire de premier degré accueille les élèves durant trois années (prima, seconda et terza media) de onze à treize ans. Il se conclut par un examen

\footnotetext{
${ }^{3}$ En ltalie, les familles peuvent décider si leur enfant suivra un cursus à 24 ou 40 heures par semaine.
} 
d'État, la Licenza media, dont l'obtention est nécessaire pour la poursuite d'études dans le secondaire supérieur et l'entrée dans la vie active. La Scuola media est conçue, comme en France, sur le modèle d'une organisation unique pour tous les élèves. A partir de quinze ans, les jeunes italiens entrent dans le secondaire du second degré ou supérieur, où s'établit une distinction entre filières générales, technologiques et professionnelles. De ce fait, la transition du secondaire de premier et second degré constitue un palier d'orientation important. La formation dans le secondaire supérieur se répartit entre formations mises en œuvre par les établissements sous tutelle de l'État et celles pilotées par les régions. Depuis la réforme Gelmini (legge 133/2008 mise en œuvre à partir de la rentrée 2010), le secondaire supérieur d'État pour ses trois filières générales, technologiques et professionnelles d'État est aligné sur une durée de cinq ans débouchant sur l'examen d'État (Esame di stato ${ }^{4}$ ). Les élèves y sont ainsi scolarisés de quatorze à dix-neuf ans. Le secondaire supérieur professionnel, piloté par les régions, a une durée de trois ans qui peut être prolongée par une spécialisation d'un an. Il ne donne pas accès à l'enseignement supérieur, sauf à faire un passage par un lycée professionnel d'État pour pouvoir décrocher l'Esame di Stato.

1.2 La dispersion scolaire en Italie : une notion large et instable, mais fondée sur l'inclusion

Le terme "décrochage » n'existe pas en italien. Le terme le plus couramment employé pour renvoyer au décrochage scolaire est celui de « dispersion scolaire » (dispersione scolastica). Il est important de clarifier cette notion parce qu'elle renvoie à une réalité plus large que l'abandon de scolarité.

En Italie, la notion de dispersion scolaire recouvre tous les mécanismes et situations d'éloignement des élèves d'un cursus normal. L'abandon scolaire en fait partie, évidemment, mais au même titre que le changement d'établissement, l'exclusion, l'absentéisme. Ainsi que le rappelle une experte des politiques de lutte contre le décrochage en Italie : «Par le terme de dispersion scolaire on entend, en Italie, un ensemble de phénomènes, comme par exemple l'abandon scolaire, l'absentéisme, le transfert d'une

- L'Esame di Stato, aussi appelé Esame di Maturitá, (examen d'État ou de maturité) est, comme le baccalauréat en France, le diplôme qui sanctionne la fin des études secondaires supérieures. 
école à l'autre, etc. Tous ces phénomènes sont considérés comme de la dispersion scolaire ».

Pour autant, il n'existe pas de définition stabilisée de cette notion de dispersion scolaire comme en témoigne cette réponse à la question « Existet-il une définition officielle de l'abandon scolaire en Italie ?», adressée au responsable du service en charge des politiques de lutte contre le décrochage scolaire au ministère de l'Instruction, de l'Université et de la Recherche (MIUR) : «Ceux qui n'ont pas obtenu un diplôme à 18 ans, c'est ça? Oui, mais à quel âge on le vérifie ? En fait, nos élèves passent l'Esame di stato à 19 ans. Je ne sais pas répondre précisément à cette question. Je ne crois pas qu'il y ait une définition légale, mais que nous avons adopté la définition européenne standard. Nous allons nous renseigner ». Plus prosaïquement, cette dirigeante d'un lycée du Latium (Istituto Savi, Viterbo) établit une distinction subtile entre l'abandon et la «mortalité » scolaire : «Par mortalité, on entend les élèves qui abandonnent l'école et ne vont nulle part ailleurs ou bien s'ils abandonnent cette école pour aller dans une autre. Il faut s'entendre sur ce qu'on entend par dispersion». Selon les interlocuteurs, la notion de dispersion scolaire renvoie ainsi à une large variété de phénomènes.

De manière générale, dans les établissements rencontrés, la notion de dispersion scolaire est étendue à tous les élèves en difficultés, que celles-ci relèvent de conditions physiologiques, psychologiques ou économico-socioculturelles. Elle englobe ainsi, en premier lieu, les élèves en situation de handicap et les élèves diagnostiqués avec des troubles spécifiques d'apprentissage (DSA). Ces deux catégories ont été élargies par une directive ministérielle de 2012 à la notion d'élèves à besoins éducatifs spécifiques (BES) signalés par leurs parents comme étant confrontés à des difficultés familiales, linguistiques ou socio-économiques entravant une scolarité sereine.

Depuis les années 70, la problématique de l'inclusion scolaire est un déterminant clé des référentiels d'action publique qui structurent le système scolaire italien (Albanese \& Pieri, 2013 ; De Anna, 2009). Elle constitue, de ce fait, un point d'entrée sur le décrochage scolaire en termes de risques, l'abandon scolaire étant l'une des conséquences potentielles de l'échec de l'inclusion scolaire.

\subsection{De la difficulté à chiffrer le décrochage scolaire}


Combien l'Italie compte-t-elle de décrocheurs ? La question est simple, et comme souvent la réponse l'est beaucoup moins. Le décomptage des décrocheurs, rendu stratégique par l'importance de ce phénomène sur les agendas politiques européen, national et régional, dépend principalement de deux éléments: la définition retenue du décrochage et les modalités techniques de recueil des informations statistiques sur l'abandon scolaire.

En termes de définition retenue du décrochage, l'Italie est confrontée à un dilemme. Celui-ci met en tension une définition extensive de la dispersion scolaire, fondée sur son référentiel national d'inclusion scolaire, et l'importance des prescriptions européennes. Depuis 2005 (Decreto Legislativo n. 76/2005), un registre national des élèves a été créé pour recueillir des données sur les élèves, et notamment sur l'abandon scolaire. Sa mise en œuvre n'a pas été substantielle pour des raisons liées aux difficultés techniques de compatibilité des bases de données territoriales et $\mathrm{du}$ fait des nombreux changements de majorité aux niveaux national et locaux (cf. infra). La loi 221/2012 a relancé le processus de constitution d'un registre national et notamment l'intégration des bases de données régionales et des établissements locaux dans un registre (Anagrafe) national.

Cette technique de comptage a toutefois été assez critiquée, notamment pour son manque de coordination entre niveaux territoriaux et établissements, comme le confirme en entretien cette chercheure italienne: "Mais si je devais parler seulement de décrochage, alors je devrais me baser sur d'autres données fournies par le ministère et qui sont basées sur les inscriptions des élèves d'une année sur l'autre. Mais comme il n'existe pas, en Italie, de code d'identification individuel pour les élèves, il n'est pas possible de suivre l'intégralité du parcours des élèves ». Et les agents du ministère de renvoyer la responsabilité aux régions quant aux difficultés à concrétiser ce projet de répertoire national ... «Ici ce sont les régions qui sont responsables de la formation professionnelle et il est difficile d'obtenir des données auprès d'elles. On est en train de construire un registre (anagrafe) national qui est élargi à la formation professionnelle alors qu'auparavant il ne concernait que l'instruction (générale). Ça permettra d'avoir des données plus complètes sur les élèves qui ne suivent pas les parcours de l'éducation nationale et paritaire» (Entretien au MIUR, Roma). 
En l'absence de définition officielle du décrochage scolaire, il est régulièrement fait référence, dans les publications officielles et dans les travaux de recherche disponibles, à l'indicateur européen ESL (Early School Leaving-Abandon scolaire précoce $)^{5}$. Le recours à cet indicateur présente un double intérêt. D'une part, il permet d'étalonner la situation italienne au regard de l'objectif de $10 \%$ d'ESL fixé pour tous les pays de l'Union européenne à l'horizon 2020, en permettant une comparaison à l'égard des autres pays de l'UE. D'autre part, il permet, par sa récurrence, une approche longitudinale des trajectoires nationales et régionales au regard du phénomène du décrochage. Dans cette perspective, le taux de décrochage scolaire relevé en 2016 (extrait de mai 2017) est de 13,8\% pour l'Italie, contre $8,8 \%$ en France, pour une moyenne européenne de 10,8 pour les 27 pays de l'UE.

\subsection{Une variable clé des politiques éducatives : la continuité de l'action publique}

Les politiques de lutte contre le décrochage scolaire s'inscrivent dans un cadre institutionnel formé par le système éducatif et son environnement. C'est une variable clé de compréhension de la formulation et de la mise en œuvre des dispositifs d'action publique. L'Italie est renommée pour son instabilité gouvernementale, que les réformes successives des institutions peinent à résorber. Il s'ensuit un questionnement légitime sur la continuité des institutions éducatives comme cadre aux politiques de lutte contre la dispersion scolaire. Au-delà de cette dimension sémantique, c'est naturellement l'organisation du ministère et son leadership qui constituent les éléments clés de la stabilité des politiques éducatives. Concernant la dimension organisationnelle, les changements de structure interne du MIUR sont fréquentes et assez peu lisibles, tout en se doublant d'un turn-over

\footnotetext{
"Cet indicateur « se réfère aux personnes âgées entre 18 et 24 ans qui satisfont aux deux conditions suivantes : a) le plus haut niveau d'éducation ou de formation qu'elles ont atteint correspond aux niveaux 0,1 ou 2 de la CITE 2011 (niveaux 0, 1, 2 ou 3C court de la CITE 1997), et b) elles n'ont pas suivi d'éducation ou de formation (en d'autres termes, ni formelle ni non formelle) dans les quatre semaines précédant l'enquête. http://ec.europa.eu/eurostat/statistics-explained/index.php/Early leavers from education and training/fr (consulté le 18/10/2017). La classification internationale type de l'éducation (CITE) est le fondement des statistiques internationales sur l'éducation et décrit différents niveaux d'éducation. La CITE 20I1 comprend neuf niveaux différents: l'éducation de la petite enfance (niveau 0), l'enseignement primaire (niveau 1), le premier cycle de l'enseignement secondaire (niveau 2), le deuxième cycle de l'enseignement secondaire (niveau 3), l'enseignement post-secondaire non-supérieur (niveau 4), l'enseignement supérieur de cycle court (niveau 5), le niveau licence ou équivalent (niveau 6), le niveau master ou équivalent (niveau 7 ), et le niveau doctorat ou équivalent (niveau 8).
} 
important des responsables de service. Ainsi, par exemple, durant les sept mois de cette enquête de terrain sur le décrochage scolaire, la direction du service en charge du décrochage scolaire (Direzione generale per lo studente, l'integrazione e la partecipazione au sein du Dipartimento per il sistema educativo di istruzione e di formazione - Direction générale des élèves, de l'intégration et de la participation au sein du Département système éducatif et de formation) était vacante. Elle l'est d'ailleurs toujours six mois plus tard.

Les changements réguliers de ministres de l'instruction constituent un grief récurrent des enseignants qui se plaignent des changements de cap incessants, chaque nouveau détenteur du portefeuille étant censé engager sa propre réforme qui portera son nom et constituera les prémisses d'une confrontation inévitable avec les enseignants et leurs syndicats. L'instabilité ministérielle est un indicateur fort de la permanence et de la continuité des politiques éducatives qui n'est pourtant jamais mobilisé comme tel. Qu'en est-il exactement? Nous avons reconstitué la généalogie des ministres italiens de l'éducation sur 30 ans - pour couvrir très largement la période où émerge et se développe la préoccupation pour la déscolarisation - afin de mesurer la longévité moyenne d'un ministre italien de l'instruction publique. Les résultats sont éloquents : la longévité moyenne d'un ministre italien de l'instruction est de moins de deux ans, soit 1,8 année (683 jours) en moyenne, pour un écart allant de 252 jours pour le mandat le plus court (Onofrio) à 1801 jours pour le plus long (Moratti) réparti sur deux gouvernements (Berlusconi II et III). Par comparaison, la longévité politique des ministres français de l'éducation est à peine supérieure, soit en moyenne de 2,2 années (810 jours). Difficile, dans un tel contexte, d'instaurer une continuité des objectifs et des moyens qui soient de nature à permettre une action publique cohérente et capable de structurer durablement l'intervention du système éducatif sur le phénomène de la dispersion scolaire.

\subsection{Des variations territoriales fortes et aux motifs divers}

Un des traits marquants de la problématique du décrochage scolaire, en Italie, tient aux fortes variations régionales. Les taux de décrochage par région révèlent en effet des inégalités frappantes qui recoupent des clivages territoriaux anciens et particulièrement vivaces. Le graphique 1 rend 
compte de ces inégalités territoriales au regard de la sortie précoce du système scolaire. Les écarts très importants vont du simple à plus du triple.

Les explications de ces variations régionales sont multiples. Deux facteurs explicatifs sont néanmoins régulièrement mobilisés par nos interlocuteurs pour expliquer les taux élevés de certaines régions : la situation économique et sociale dégradée pour ce qui concerne les régions méridionales ; les effets d'aspiration des élèves par le tissu entrepreneurial des PME/PMI (petites et moyennes entreprises/industries) avant leur diplomation dans l'Italie du Centre-Nord-Est (généralement désigné par l'expression de Troisième Italie).

En perspective longitudinale, on constate pourtant, sur dix ans, une baisse générale de la population des sortants précoces (Early School Leavers) dans toutes les régions. Cette baisse est significative puisque, globalement, la situation italienne comparée à la moyenne des pays de l'Union européenne s'est améliorée. Ainsi en 2006, l'Italie avec un taux d'abandon précoce de $20,4 \%$ se situait à cinq points au-dessus de la moyenne européenne $(15,4 \%)$. En 2016, avec un taux de 13,8 \%, elle n'est plus qu'à trois points de la moyenne communautaire $(13,8 \%)$ et proche de l'objectif de $10 \%$ pour 2020 .

\section{Graphique $n^{\circ} 1$ : Taux de sortants précoces par région}




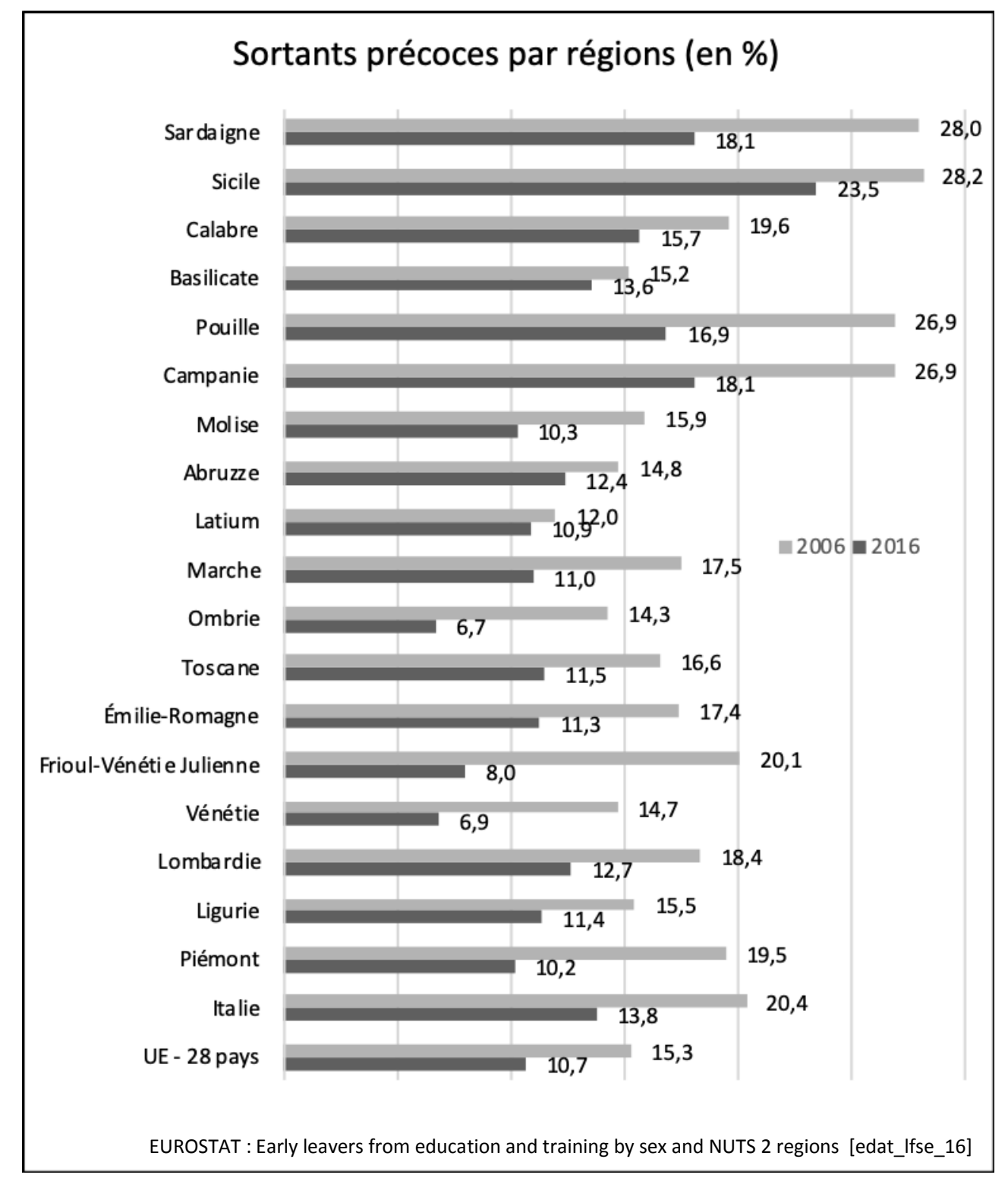

Comprendre cette diminution suppose d'étudier l'organisation de la lutte contre l'abandon scolaire en Italie. C'est l'objet de la partie suivante. 


\section{Les politique de lutte contre le décrochage scolaire en Italie : une étude en région Lazio}

Le Latium (Lazio) est une région centrale de l'Italie. Cette région a pour centre administratif et politique la métropole de Rome, mais ne s'y réduit pas. La région est vaste, $17207 \mathrm{~km}^{2}$, peuplée de presque six millions d'habitants et très diverse dans ses paysages. Des côtes aux cités balnéaires, des zones de montagne pour un quart de son territoire, des espaces ruraux rythmés par les collines de tuf et des villes moyennes, c'est un espace humain et politique contrasté et intermédiaire qui confine à la Toscane au nord et à la Campanie au sud.

L'enseignement secondaire y accueille un peu plus de 400000 élèves. Au regard de la question du décrochage scolaire, elle fait partie des régions intermédiaires ayant atteint récemment l'objectif des $10 \%$ de sorties précoces.

Cette partie consacrée à l'analyse des politiques publiques de lutte contre la dispersion scolaire aborde les questions de la mise à l'agenda de cette problématique et de l'offre publique d'intervention/remédiation étudiée à deux niveaux, celui du design des politiques italiennes et celui des instruments mis en œuvre dans les établissements scolaires.

L'analyse de l'offre publique n'aborde pas en tant que tel, et faute de données évaluatives probantes, la question de l'efficacité et de l'impact de ces dispositifs sur les taux de décrochage. Le caractère récent et très décentralisé des politiques italiennes explique cette difficulté analytique. Leur mise en œuvre explique, sans doute pour partie, la baisse rapportée ( cf supra) des taux de décrochage sans qu'un lien de causalité robuste et circonstancié puisse être identifié. On se limitera donc ici à l'identification de cette offre via les instruments d'action publique développés.

\subsection{Le processus de mise à l'agenda : une brève chronologie}

Le processus de mise à l'agenda politique du problème social du décrochage scolaire intervient de manière assez tardive, en dépit de quelques tentatives commençant au milieu des années 90.

Le graphique 2 présente les moments et instruments clés concernant la mise à l'agenda du décrochage scolaire. 


\section{Graphique 2. Frise chronologique de la mise à l'agenda du décrochage scolaire en Italie}

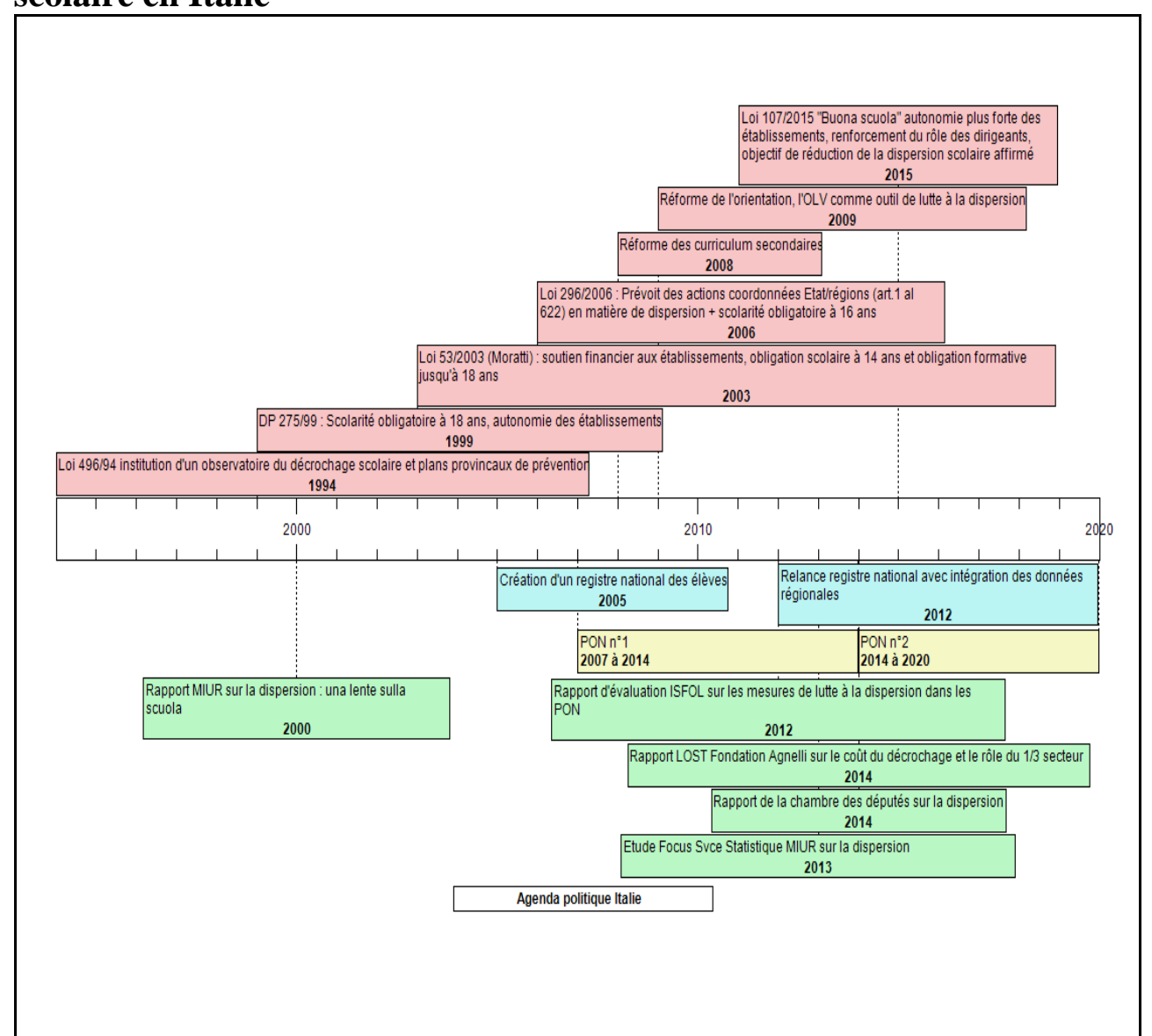

Pour une meilleure compréhension de ce graphique : les PO (Programmes Opérationnels N pour national $\mathrm{R}$ pour régional) constituent la déclinaison des financements européens en plan d'action. L'ISFOL (Istituto per lo sviluppo della formazione professionale dei lavoratori) est un organisme public d'étude et de statistiques sur le système de formation et d'emploi italien. Il est devenu, depuis 2016, l'INAPP (Istituto Nazionale per l'Analisi delle Politiche Pubbliche).

\section{Source : Auteur.}

Au-delà de sa chronologie factuelle, la mise à l'agenda politique d'une question sociale repose sur une série de mécanismes cognitifs, de stratégies d'acteurs et de rapports de pouvoirs, dont l'élucidation est nécessaire pour comprendre les dispositifs d'action publique et leurs modalités de mise en 
œuvre. Du point de vue des rares travaux scientifiques ${ }^{6}$ sur le sujet, le décrochage scolaire constituait, de manière globale «a neglected problem for the social and political agenda in Italy due to its endemic nature and to the scarce pressure by the public opinion and the media $^{7}{ }_{\text {(Colombo, 2013, }}$ p. 11). Il faut attendre le début des années 2010 pour que le thème du décrochage trouve une résonance sur l'agenda politique national, comme le rappelle en entretien cette chercheure italienne : "Jusqu'à au moins 2013, cette question n'a pas été prioritaire sur l'agenda gouvernemental. A partir de 2013, il y a ce décret-loi 104/2013 où s'est immiscée, à l'article 7, la lutte contre la dispersion scolaire comme une mission fondamentale de l'école. Jusqu'en 2013, la dispersion scolaire a été un thème parmi d'autre sur l'agenda politique, mais sans relief particulier ».

Comme dans nombre de pays, la prise de conscience de la problématique de l'abandon scolaire tient en premier lieu à la production de données sur le phénomène (MIUR, 2000 et 2006). C'est au cours des années 2000, et dans le cadre de la programmation des fonds européens, que l'Italie va ainsi prendre conscience de l'importance du phénomène de la dispersion scolaire, comme le rappelle cet administrateur du Ministère de l'instruction publique : "En Italie, il n'y a pas eu d'évènements déclencheurs violents comme les émeutes en France. Je crois qu'on en a pris conscience après 2004, quand sont sorties les premières données sur la dispersion qui était très élevée dans certaines régions $d u$ sud de l'Italie. On a commencé à prendre conscience du phénomène, mais les politiques publiques ont été plus lentes à se mettre en place » (Entretien MIUR, Roma). Une série de documents atteste de l'importance croissante prise par la question de la dispersion scolaire sur l'agenda politique italien, en lien avec la production de données sur le décrochage au début des années 2010. Un rapport parlementaire de 2014 signale ainsi que ce problème a pris une «importance sans précédent $\gg$ (Camera dei deputati, 2014, p. 5). Parmi les publications relatives à la problématique de la dispersion qui fleurissent entre 2010 et

\footnotetext{
${ }^{6}$ Les travaux scientifiques sur le décrochage scolaire constituent une littérature embryonnaire largement dominée par des ouvrages pratiques. Peu de travaux abordent la question des politiques de lutte contre le décrochage d'où se dégagent ceux conduits par Maddalena Colombo à l'université de Milan. Pour un exemple présenté à la conférence de comparaison internationale du CNESCO en novembre 2017: http://www.cnesco.fr/wp-content/uploads/2017/11/p2_maddalena_colombo.pdf (consulté le 23/08/2019).

7 «Un problème négligé sur l'agenda social et politique en Italie en raison de son caractère endémique et de la faible pression de l'opinion publique et des médias » (traduction par l'auteur)
} 
2016, se mêlent des études produites par les autorités publiques et par des fondations privées ${ }^{8}$.

L'inscription récente de la dispersion scolaire en haut de l'agenda sectoriel des politiques éducatives (Cobb \& Elder, 1975) s'est accompagnée d'une transformation de son mode de légitimation. En plus des dimensions territoriales européenne comme régionale, elle s'adosse aujourd'hui fortement à l'importance de l'enjeu migratoire. L'élévation de la population migrante et la médiatisation très importante des flux de réfugiés constituent en effet un deuxième point d'ancrage plus récent de la grammaire de justification des politiques de lutte contre la dispersion scolaire. «Et puis, durant ces 10 dernières années au moins, il y a eu ce phénomène de fort processus migratoire et le pourcentage d'élèves étrangers est devenu très important et le phénomène de la dispersion s'est aggravé de ce fait. (...) Pour nous, l'immigration est un phénomène récent et qui s'est fortement développé. Cela a créé des problèmes pour les écoles parce que tous les élèves sont accueillis, qu'ils soient réguliers ou non, cela a créé de gros problème d'accueil et le pourcentage de décrochage parmi les élèves nonitaliens est très élevé » (Entretien MIUR, Roma). L'étude statistique conduite par le MIUR, en 2016, atteste de l'importance numérique et de l'enjeu que représente l'intégration des élèves d'origine étrangère. L'augmentation de la part de ces élèves dans les écoles italiennes est en effet particulièrement significative, puisqu'elle est passée de $0,6 \%$ en 1995/96 (50 322 élèves) à 9,2\% pour l'année scolaire 2015/2016, soit 814851 élèves de nationalité non-italienne sur un total de 8826893 élèves scolarisés cette année-là (MIUR, 2017a).

La migration comme mécanisme causal de la dispersion scolaire est également régulièrement évoquée dans les entretiens avec les autorités publiques, qu'elles relèvent du ministère, de l'équivalent fonctionnel régional des rectorats (l'Ufficio scolastico regionale, USR Lazio) ou du Conseil régional. «Récemment, en juillet, l'appel d'offre 'Scuola al centro'

\footnotetext{
${ }^{8}$ Parmi les premières, on notera le rapport de L'ISFOL (Istituto per lo sviluppo della formazione professionale dei lavoratori cf. note 4) sur les mesures mises en œuvre dans le cadre du programme opérationnel national des fonds structurels (ISFOL 2012); le Focus du service statistique du MIUR (MIUR, 2013), ainsi que l'extraction italienne du rapport Euridyce sur l'abandon précoce (MIURINDIRE, 2014) ou le rapport INDIRE sur les réseaux et les pratiques innovantes en matière de lutte contre la dispersion (INDIRE, 2016). Parmi les secondes, on relèvera le rapport de la Fondation Brodolini sur le projet RIDISCO dans la province de Rome (Fondazione Giacomo Brodolini, 2007), l'étude conduite par l'ONG Save the children sur le projet InContro, ainsi que celle conduite sous l'égide de la Fondation Agnelli sur le coût du décrochage et le rôle du tiers secteur (LOST, 2014).
} 
(l'école au centre) vise les quatre villes qui font face à une incidence majeure d'élèves étrangers et des écoles de quartiers périphériques. (...) Les fonds issus du CNCL (Contratto Collettivo Nazionale di Lavoro Contrat National Collectif de Travail-cf. note 14 - permettant le financement d'heures complémentaires pour les enseignants) sont divisés en 2 : zones à forts processus migratoires (pourcentage supérieur à $15 \%$ d'élèves étrangers) et zones ou le taux d'abandon ou de redoublement sont élevés. Nous connaissons les écoles qui se trouvent dans ces zones, elles sont recensées dans une liste d'établissements éligibles » (Entretien USR Lazio, Rome). L'écho de cette mise en relation décrochage/migration se fait encore plus fort au niveau des établissements. Les chefs d'établissements rencontrés, que ce soit au centre-ville de Rome, en périphérie ou dans les zones rurales de la région, établissent tous ce lien. «Ceci dit, ces dernières années, nous avons eu une augmentation des élèves étrangers de première génération qui ne parlent pas italien. Ils ne sont pas nombreux, sept ou huit, mais ils ont de grosses difficultés parce qu'ils ne maîtrisent pas la langue » (Istituto Savi, Viterbo); "Et puis, il y a évidemment l'autre grand problème qui est celui de la migration et donc il y a une partie de la population italienne concernée par les phénomènes de migrations, les étrangers qui arrivent en Italie, plus particulièrement au nord, parce qu'il y a plus de possibilités d'emploi. Là on peut avoir des problèmes de dispersion liés à des dimensions culturelles et économiques surtout » (Liceo Virgilio, Rome).

Ce lien est ainsi nettement établi à tous les niveaux de l'institution scolaire italienne. Pour autant, cette relation causale est-elle solidement établie ? Rien n'est moins sûr. Un premier élément global incite à la prudence. En effet, la population migrante a doublé au cours des dix dernières années, alors même que les taux de sorties précoces sans qualification baissent de manière sensible. On constate également que les élèves de nationalité étrangère tendent à se concentrer dans le nord du pays, alors que le décrochage sévit le plus fortement dans le sud. Les cinq premières régions où la proportion d'élèves étrangers est la plus élevée sont ainsi, par ordre d'importance, l'Émilie-Romagne, la Lombardie, l'Ombrie, la Toscane et la Vénétie) alors que les cinq régions présentant les taux de décrochage les plus élevés sont des régions du Sud de l'Italie (Sicile, Sardaigne, Campanie, Pouille, Calabre). Précisons enfin que les élèves étrangers optent plus souvent que les élèves italiens pour des formations professionnelles et techniques qui concentrent les taux les plus élevés d'élèves en difficultés. Néanmoins, en l'absence de données fiables sur les taux de décrochage par 
nationalité, un indicateur permet de souligner la réalité des difficultés d'insertion scolaire et professionnelle des jeunes d'origine étrangère, c'est celui des NEETs $^{9}$ (les jeunes de 15 à 24 ans qui ne sont ni en emploi ni en scolarité initiale ou continue). Parmi ces jeunes inactifs ou au chômage, les étrangers sont surreprésentés de dix points par rapport aux jeunes italiens en moyenne; la situation des jeunes femmes apparaît particulièrement préoccupante et probablement liée à des facteurs qui dépassent la simple question de la scolarisation.

Tableau 1. NEETS (15-24 ans) répartition par nationalité et sexe en 2012 (en \%)

\begin{tabular}{|l|l|l|l|}
\hline & Italiens & Étrangers & Total \\
\hline Filles & 19,1 & 38,2 & 21,0 \\
\hline Garçons & 20,8 & 23,9 & 21,2 \\
\hline Total & 20,0 & 30,8 & 21,1 \\
\hline
\end{tabular}

Source : Italia Lavoro (enquête Force de travail, ISTAT)

NEETS : Not in Education, Employment or Training (Ni en éducation, en emploi et en formation)

2.2 Les politiques publiques de lutte contre le décrochage scolaire : une autonomie des établissements conditionnée par le financement sur projets

Il importe de préciser, en préambule, que les politiques italiennes de lutte contre le décrochage scolaire se concentrent sur le champ de la prévention/intervention, en l'absence d'une offre de remédiation comparable à celle proposée, par exemple, par le réseau des missions locales françaises. De manière globale, la mise en œuvre l'objectif de lutte contre le décrochage fixé par la loi de 2015, repose sur l'autonomie d'action des établissements et le rôle prépondérant de leurs dirigeants.

Le rôle du ministère via les USR tient à la définition de grands objectifs et à la distribution des ressources financières. Interrogés sur le rôle du MIUR dans la lutte contre la dispersion scolaire, ce responsable rappelle comment se répartissent les tâches: "Le ministère donne les lignes directrices

\footnotetext{
${ }^{9}$ Rappelons ici que la population des NEETs (Not in Education, Employment or Training) ne recouvre que partiellement celle des jeunes en situation de décrochage et qu'elle inclut, par exemple, des jeunes ayant obtenu un diplôme de fin d'études secondaires.
} 
générales et les offices régionaux ont leur autonomie. L'USR Sicile et celui du Piémont ont des politiques différentes. Le ministère donne juste des grandes lignes d'intervention et ensuite, les USR élaborent leurs propres politiques publiques sur le territoire en s'inspirant des lignes directrices fournies par le ministère » (Entretien MIUR, Roma). L'administration centrale se contente donc de définir des objectifs généraux et de mettre des fonds à disposition des établissements, tout en renvoyant leur mise en œuvre concrète aux territoires. Il n'est donc pas étonnant que les financements structurels de l'Union européenne, principal bailleur de fonds, structurent de manière décisive cette politique. "L'impulsion à lutter contre la dispersion est venue de l'Europe avec les ressources qu'elle a données, il y a eu des interventions plus structurelles à travers la programmation 2000-2006. Mais concentrées dans le sud de l'Italie, parce que les deux précédentes programmations 2000-2006 et 2007-2013 prévoyaient l'accès aux financements seulement pour les écoles du sud de l'Italie»(Entretien MIUR, Roma).

Ainsi le PON ${ }^{10}$ 2000-2006 (Scuola per lo sviluppo - L'école pour le développement) a promu des actions de prévention et de remédiation dans les régions méridionales. La programmation suivante (2007-2013 - 270 millions d'euros) cible les interventions sur les quatre régions de l'objectif convergence (Campanie, Calabre, Pouille, Sicile). Une action complémentaire (42 millions d'euros), mise en place dans le cadre du Plan d'Action Cohésion engagé en 2012, visait à soutenir la mise en œuvre de réseaux d'établissements dans les régions Convergence. La programmation 2014-2020 se démarque par un ciblage territorial qui se déplace des régions méridionales vers l'identification de zones à risque. C'est sur cette base en effet qu'est mis en œuvre le programme sur projets «Scuola al Centro » ciblé dans une première phase expérimentale, en 201,6 sur les quartiers en difficultés de quatre métropoles italiennes - Rome, Milan, Naples et Palerme - puis élargi à une base nationale à partir de l'année scolaire 20162017. Il a été doté de 187 millions d'euros sur financements communautaires. Cet appel à projet vise à développer les activités extracurriculaires et les plages d'ouverture des écoles l'après-midi pour des activités de lutte contre la dispersion. Il a bénéficié à 4633 écoles. A ces différents éléments de financements communautaires, il faut rajouter le plan extraordinaire 2014, initié par le décret ministériel 104 de 2013. Ce plan

${ }^{10}$ Cf. supra note $n^{\circ} 4$. 
affecte 15 millions d'euros aux établissements secondaires pour soutenir des actions de lutte contre la dispersion et développer les stages professionnalisants. Enfin, pour clôre ce rapide survol des interventions nationales en matière de lutte contre le décrochage, il faut signaler le rôle joué par l'article 9 du CCNL (Contratto Collettivo Nazionale di Lavoro) ${ }^{11}$. Sur la base de cet article, le ministère affecte aux écoles des zones à risque, via les USR, des ressources destinées à financer les heures des enseignants s'engageant dans des projets visant à lutter contre la dispersion. Au final, il apparaît clairement que le rôle des politiques à un niveau national est orienté avant tout vers la mise à disposition des établissements de ressources financières distribuées sur projet.

Qu'en est-il du niveau régional ? Les USR (Ufficio Scolastico Regionale) ont-ils une fonction plus structurante que le ministère ? Clairement, la réponse est négative. Les USR constituent un relai des orientations et des financements ministériels, une structure gestionnaire avant que d'être politique. «Nous sommes un centre de gestion, nous gérons les fonds. Nous avons une fonction de support et d'interface entre le ministère et les institutions d'enseignement de manière à ce que ces fonds soient dépensés de manière cohérente par rapport aux finalités des différents décrets ministériels qui mettent ces fonds à disposition» (Entretien USR Lazio, Rome).

Au niveau régional également, les conseils régionaux représentent un autre acteur de la lutte contre le décrochage scolaire. Dans une forme de subsidiarité inversée, les régions interviennent auprès des élèves au gré de leurs moyens et en complémentarité avec les obligations qui lient un État à ses jeunes. C'est du moins ce que rappelle d'emblée, lors d'un entretien, la chargée de mission du Conseil régional Lazio «On prend en compte la demande des jeunes, sachant que le système n'est pas extensible à l'infini comme dans l'éducation nationale où l'État a l'obligation d'accueillir tous les jeunes, la région accueille autant de jeunes que possible ». Cela dit, les

\footnotetext{
${ }^{11}$ En Italie, les CCNL jouent un rôle de cadrage des conditions d'emploi assez similaire à celui des conventions collectives en France. Il concerne les salariés des secteurs public comme privé. Celui du MIUR, qui n'a pas été renouvelé depuis la version 2006-2009, mais simplement reconduit à l'identique, comprend, à son article 9 une disposition concernant les zones à risque «misure incentivanti per progetti relativi alle aree a rischio, a forte processo immigratorio e contro l'emarginazione scolastica » (Mesures d'incitation en vue de projets relatifs aux zones à risque, à fort processus migratoire et contre la marginalisation scolaire).
} 
régions, ou du moins celle du Latium concernée par cette étude ${ }^{12}$, interviennent principalement sur deux registres: la formation professionnelle et le financement sur projets. Ce sont en effet les régions italiennes qui pilotent les Centres de formation professionnelle et qui délivrent des qualifications professionnelles à l'issue d'une formation de trois ans. Par ailleurs, les conseils régionaux interviennent, comme l'État, sur le décrochage, en finançant des projets au sein des établissements. C'est le cas notamment du projet «Fuoriclasse » mis en œuvre dans le cadre de la programmation 2014-2020 du POR (Programma Operativo Regionale) cofinancé là encore par le FSE (fonds social européen). Doté de 1,5 millions d'euros, ce programme constitue une manne supplémentaire pour les établissements qui demeurent, en tout état de cause, au centre de la lutte italienne contre la dispersion scolaire.

On l'aura donc compris, le cœur opérationnel des politiques de lutte contre le décrochage scolaire en Italie réside dans les établissements. Depuis la mise en œuvre d'une politique d'autonomisation et de responsabilisation sans cesse plus large des établissements, les acteurs régionaux et nationaux interviennent essentiellement sur un registre cognitif et financier. Ce sont les établissements qui portent la responsabilité de trouver des réponses opérationnelles au problème du décrochage.

\subsection{Des dispositifs congruents mis en œuvre dans les établissements}

Le niveau de la mise en œuvre des politiques de lutte contre la dispersion scolaire, en Italie, est ainsi celui de l'école. C'est le principe de l'autonomie croissante des établissements qui donne corps et légitimité à leur rôle central. Pour autant, deux constats liés l'un à l'autre s'imposent. Le premier tient à l'innovation ou la démarcation des actions entreprises d'un établissement à l'autre. Il ressort des entretiens conduits et de l'analyse documentaire que les facteurs de différenciation entre établissements sont relativement faibles s'agissant des mesures de lutte contre la dispersion scolaire. On retrouve ainsi, régulièrement, une série de mesures communes (cours de rattrapage, activités poméridiennes, modules et cellules d'aide, mesures linguistiques, etc.) à l'ensemble des établissements. L'hypothèse d'une forte

\footnotetext{
${ }^{12}$ Pour une étude des politiques régionales de lutte contre le décrochage dans deux autres régions (Pouilles et Lombardie), on verra la thèse d'Elisabetta De Feudis (De Feudis, 2017).
} 
différenciation liée à l'autonomie des établissements est ainsi infirmée, au moins sur la zone d'enquête considérée, le Latium.

L'explication de cette faible différenciation nous conduit à un second constat. En dépit d'une forte autonomie organisationnelle des établissements, le rôle intégrateur joué par les mécanismes financiers semble prégnant. En effet, les actions mises en œuvre par les établissements sont financées de manière quasi exclusive par des subventions obtenues dans le cadre de la réponse à des appels d'offre nationaux et régionaux eux-mêmes abondés par des fonds européens. La nature des appels d'offre et l'instruction qui en est faite par les différents donneurs d'ordre (Ministère, USR ou Conseils régionaux) semblent ainsi structurer les dispositifs concrets autour de quelques grandes catégories et types d'action que nous allons à présent explorer.

Logiquement, le premier instrument de lutte contre le décrochage scolaire, en Italie, est constitué par la recherche de moyens financiers pour soutenir les actions dans les établissements, grâce à l'Union européenne. "Nous participons depuis l'année dernière, grâce au seigneur, nous avons remporté beaucoup d'appels d'offre, surtout sur les PON, les fonds européens. Avec eux, nous avons refait tout le réseau Wifi ici, comme à Castelforte » (Entretien Istituto Omnicomprensivo di Castelforte). C'est la formule des appels d'offre du ministère et de son antenne régionale, l'USR, qui est la plus fréquemment mobilisée par les établissements «Plus ou moins, il y a chaque année un appel d'offre sur le thème de la lutte contre l'abandon scolaire. Nous présentons un projet, s'il est approuvé, ils nous donnent des fonds avec lesquels nous mettons en place des formes de soutien aux élèves » (Entretien Liceo Majorana, Roma).

Les financements régionaux constituent une autre source possible. Ainsi, le volet $\mathrm{B}$ du projet «Fuoriclasse » du Conseil régional Lazio ${ }^{13}$ vise à réduire le décrochage scolaire en finançant une large gamme d'actions. Deux établissements parmi ceux rencontrés émargent à ce fonds pour leurs actions qu'ils décrivent ainsi : "C'est un appel d'offre auquel on peut présenter des projets, c'est ce que nous avons fait pour la visite à Auschwitz il y a quelques années»(Entretien Liceo Majorana, Roma); «Aujourd'hui, les élèves de Castelforte sont ici à Gaeta parce que nous avons participé à l'appel à projet 'Fuoriclasse' et on a gagné. L'objet de ce projet était la représentation cinématographique de la légalité et la rencontre avec des

${ }^{13}$ http://www.progettofuoriclasse.it 
magistrats et acteurs sur le thème de la légalité. Mais pour les élèves, c'est gratuit. On a participé à cet appel à projet pour leur donner la possibilité de sortir un peu du village» (Entretien Istituto Omnicomprensivo di Castelforte).

L'étude évaluative du ministère de l'éducation relative à la mise en œuvre de la programmation 2014-2020 montre un taux d'adhésion élevé puisque plus d'un établissement sur deux $(53,8 \%)$ a participé au PON. Là encore, les taux varient très fortement au profit des régions méridionales. La région Lazio a un taux d'adhésion de 45,4\% (MIUR, 2017b). Mais, en tout état de cause, l'accès à ces financements sur projet est intimement lié à la capacité de la direction des établissements à soutenir une ingénierie de projet. A titre d'exemple, la vacance de direction au sein de l'un des instituts techniques enquêté s'est immédiatement traduite par une baisse conséquente des financements disponibles pour les actions de lutte contre le décrochage.

Parmi les dispositifs les plus fréquemment évoqués dans les établissements, les modules sous forme de cellules d'appui (sportelli) reviennent très fréquemment. Ils apparaissent clairement liés à l'instrumentation de l'inclusion scolaire. "En général, les écoles italiennes ont presque toujours au moins un expert qui est disponible, on l'appelle 'sportello di ascolto', (Cellule d'écoute) parce que ce n'est pas une relation psychologique de traitement, mais c'est un service que nous trouvons très important parce que le psychologue peut arriver à interpeller les profs à propos du cas d'un élève. C'est très important parce que les élèves peuvent choisir de parler avec l'expert plutôt qu'avec les parents ou les profs parce que c'est quelqu'un qui est hors du système et qui peut assumer une médiation » (Entretien Liceo Virgilio, Roma). Ces modules sont proposés par l'équipe enseignante et la direction aux élèves en difficultés qui peuvent s'en saisir sur la base du volontariat. Il s'agit le plus souvent d'une cellule d'écoute psychologique ou d'aide, mais elle peut également fonctionner comme un outil visant à réorienter les élèves en difficultés vers une filière plus adéquate. "Nous avons un "sportello » d'écoute psychologique pour les élèves où ils se rendent par eux-même et qui nous sert aussi pour l'orientation. De cette manière, nous cherchons, là où c'est nécessaire, à les réorienter » (Entretien Istituto Savi, Viterbo). 
Connu en France dans le cadre de la loi de refondation de l'école sous l'expression «plus de maîtres que de classes ${ }^{14}$, la pratique des enseignants surnuméraires, répandue en Europe du nord, s'est également installée dans le paysage éducatif italien avec la loi 107 de 2015. «Avec la loi 107, nous avons eu des 'docenti del potenziamento' (enseignants surnuméraires). Les établissements ont des enseignants en plus qu'ils peuvent utiliser pour des activités de projet, remplacer des enseignants absents et je dois dire que nous en utilisons une partie pour faire du rattrapage l'après-midi » (Entretien Istituto Savi, Viterbo). En lien avec l'autonomie des établissements, ces enseignants s'engagent dans des activités transversales ou de soutien aux élèves autant qu'aux enseignants ordinaires. " Ce sont des enseignants qui nous aident à gérer quotidiennement l'école, ils peuvent intervenir en cours, faire des cours de rattrapage pour les élèves en difficultés ou remplacer des enseignants manquants »(Entretien Liceo Majorana, Roma)

Mais les activités qui reviennent le plus souvent dans les entretiens sont les activités poméridiennes. Les rythmes scolaires pratiqués en Italie sont en effet concentrés sur la matinée et laissent les élèves libres en début d'aprèsmidi (généralement à 14h). Il est donc apparu logique aux établissements d'en profiter pour offrir des formules de consolidation des acquis ou de rattrapage des difficultés scolaires. Ces activités de l'après-midi sont en effet soutenues par les autorités publiques ${ }^{15}$. L'ensemble des établissements rencontrés dans le cadre de cette enquête offrent donc des activités de ce type à leurs élèves.

Pour autant, ces actions se heurtent à un problème massif, incontournable, lié à la mobilité des élèves. Les lycées de centre-ville, dont l'aire de recrutement est souvent très retreinte et localisée, ne connaissent pas ce

\footnotetext{
${ }^{14}$ Ces enseignants ont depuis été généralement repositionnés sur le dispositif de diminution des effectifs en CP (conseillers professionnels), décidé par le gouvernement Macron et son ministre de l'Éducation Jean-Michel Blanquer.

${ }^{15}$ Le décret-loi du 12 septembre 2013 introduit, à l'article 7, un élargissement des heures d'ouverture des écoles pour prévenir la dispersion scolaire. La loi Buona Scuola (Loi dite Bonne École du gouvernement Renzi) (107/2015) prévoit également l'ouverture poméridienne des écoles comme instrument de lutte contre le décrochage. Ces dispositions législatives sont relayées dans les appels d'offre et par les fonds disponibles dans le cadre de l'article 9 du CCNL (Contratto Collettivo Nazionale del lavoratori, cf. supra) destinés à offrir l'assiette financière (notamment la rémunération des enseignants) pour ces actions.
} 
problème. Mais dès que l'on quitte les grands centres-villes et qu'on gagne la périphérie ou les villes moyennes, les récriminations pleuvent. "Les inconvénients relèvent des transports qui contraignent les activités d'aprèsmidi pour pouvoir avoir un nombre suffisant d'élèves, parce qu'après 14h, les liaisons avec les autres villes sont compliquées » (Istituto Fermi, Gaeta). «A Castelforte, les projets extracurriculaires on n'en fait quasiment pas parce que les élèves ne participent pas aux activités d'après-midi. A Castelforte, à 14h, les élèves veulent rentrer chez eux» (Entretien Istituto omnicomprensivo Castelforte). Ce problème de mobilité qui s'impose pour les activités poméridiennes vaut d'ailleurs également pour les activités proposées durant les vacances scolaires.

La tentation est alors forte de se rabattre sur les moments où les élèves sont dans les établissements pour conduire des activités relevant de l'intervention ou de la prévention. Mais cette stratégie didactique n'est pas sans défaut elle aussi. Les horaires étant condensés sur un temps matinal, introduire des cours de rattrapage signifie souvent retirer les élèves en difficultés de leur classe pour leur proposer des activités de remise à niveau, mais pendant ce temps, leur classe «normale » continue ses cours que dès lors ils ratent. Cela crée une sorte de cercle vicieux pétri de bonne volonté où, pour combler les lacunes, on en crée d'autres. «De nombreuses tentatives ont été faites, comme par exemple des cours de rattrapage sur le temps scolaire. Par exemple, je fais de la mécanique, mais comme ces élèves ont besoin de cours de langue pour me comprendre, on va les retirer pendant deux heures pour des cours de langue et donc ils manquent mon cours »(Entretien Istituto Cattaneo-Testaccio).

La dernière variante d'actions en lien avec les temporalités scolaires relève des cours du soir. Le plus souvent, c'est en effet une mesure de remédiation pour des élèves déjà en situation de décrochage. Comme l'indique joliment ce chef d'établissement : "De nombreux élèves qui ne réussissent pas, qui ont eu des difficultés, qui ont connu plusieurs redoublements s'inscrivent aux cours du soir et ainsi nous les récupérons. Ils restent dans cette école; on peut leur dire que s'ils ne réussissent pas, après 18 ans, ils peuvent s'inscrire aux cours du soir. Ceux que nous perdons le matin, nous les récupérons le soir! (Entretien Istituto Savi, Viterbo)

Une série d'actions sont également conduites, visant à agir sur le climat scolaire, la formation des enseignants, les relations entre pairs ou l'accès à la culture. Parmi les actions conduites sur ce registre, on relèvera la 
formation continue des enseignants. Rendue obligatoire par la loi Buona Scuola de 2015, la formation continue des enseignants est essentiellement externalisée et fonctionne sur un système de voucher (bon d'échange). En lien avec les actions de formation continue qu'elles visent souvent à renforcer, tous les établissements conduisent également une réflexion sur les manières de faire évoluer les pratiques pédagogiques afin d'améliorer l'inclusion scolaire, notamment parce que le taux de dispersion représente aujourd'hui un indicateur dans l'évaluation des établissements et de leurs dirigeants.

Parmi les actions conduites en matière de lutte contre le décrochage, en Italie comme dans de nombreux pays, les pratiques culturelles occupent une place importante. Parfois, elles prennent la forme d'un parcours intégré, comme dans ce grand établissement du centre de Rome où l'offre culturelle, notamment patrimoniale, est gigantesque. Dans des environnements moins dotés en ressources patrimoniales et artistiques - encore qu'il faille relativiser la notion de désert culturel dans un pays débordant de sites qu'ils soient ou non valorisés - on se rabat sur des ressources plus internes où le numérique et la musique occupent des places de choix.

Pour finir sur les dispositifs internes aux établissements, on relèvera les pratiques de mentorat/tutorat. Elles visent à s'appuyer sur les élèves les plus avancés, que ce soit en niveau scolaire ou en âge, pour aider les plus jeunes ou les plus en difficultés. Ces actions, basées sur la proximité générationnelle, sont généralement vantées par les établissements qui les mettent en œuvre pour leur double effet. D'une part, l'aide est plus facilement acceptée venant d'un pair et d'autre part, elle crée, chez les protagonistes, un sens de la solidarité valorisé.

Pour clore ce tour d'horizon des actions concrètes mises en œuvre par les établissements, mentionnons rapidement les actions entreprises en direction des familles. Pourquoi une mention rapide? Non pas parce qu'elles ne seraient pas importantes ou efficientes, mais tout simplement parce qu'elles sont de loin celles qui sont le moins souvent évoquées par les interlocuteurs rencontrés. C'est un vrai paradoxe d'ailleurs, parce qu'autant on porte souvent le blâme de la difficulté ou de l'abandon sur la tête des familles défaillantes, autant c'est rarement auprès d'elles que l'on cherche des solutions !

\section{Conclusion}


Quelles sont, pour terminer, les lignes de forces qui se dégagent de cette étude ? Question ancienne, mais problème public très récent à l'agenda politique et scientifique, le décrochage scolaire devient un enjeu important, sous la pression des politiques européennes et l'objectif fixé de $10 \%$ de décrocheurs en 2020.

Ces politiques sont construites en lien avec une conception ancienne de l'inclusion scolaire, pensée dans les années 70, au regard des enfants en situation de handicap. Ce lien se matérialise, en Italie, avec la notion de dispersion scolaire qui prédomine dans le discours officiel et de terrain. Il inclut ainsi de fait l'abandon scolaire dans une perspective plus globale intégrant les élèves en situation de handicap, souffrant de troubles d'apprentissage, les élèves à besoins spécifiques et ceux victimes de harcèlement à l'école.

La lutte contre le décrochage scolaire ne fait pas l'objet d'une stratégie coordonnée par les autorités publiques, et notamment par le ministère de l'Instruction. Le mouvement d'autonomisation des établissements scolaires conduit à un pilotage central très lâche qui transite essentiellement par deux canaux : la définition de grands objectifs stratégiques et le financement par appel d'offre. Au niveau territorial, les relais sont faibles. Les USR (Uffici Scolastici Regionali) comme les conseils régionaux sont des acteurs de second rang. Les premiers relaient la mécanique ministérielle avec une faible marge de reconfiguration régionale. Les seconds ne disposent que de leur compétence sur la formation professionnelle, mais qui se joue essentiellement via les centres de formation que les régions pilotent et qui ne sont pas une ressource consistante dans la lutte contre décrochage. Le seul outil d'influence significatif est constitué par les POR (Programme Opérationnel Régional), déclinaison régionale des PON (Programmes Opérationnels nationaux) européens, qui permet aux régions d'instiller du changement par appels d'offre.

C'est finalement au niveau des établissements que se joue une lutte contre le décrochage centrée sur la prévention et l'intervention, en l'absence d'une offre de remédiation suffisante. Aujourd'hui, elle participe de l'évaluation des établissements et de leurs dirigeants qui disposent, en Italie, du fait de l'autonomie, d'un pouvoir d'action grandissant. Cette mécanique essentiellement fondée sur la capacité des établissements à remporter des appels d'offre pour financer leurs actions est aléatoire. Elle suppose une mobilisation des équipes pédagogiques et administratives qui semblent s'épuiser dans cette course aux euros et où les disparités de forces en 
ingénierie de projet font la différence. Pour autant, on constate que l'autonomie croissante des établissements leur fournit des clés pour adapter leur offre aux besoins du territoire et par là pouvoir s'appuyer sur des ressources externes pour contrer l'abandon scolaire. Néanmoins, cette inscription des écoles italiennes dans leur territoire ne semble pas se traduire, comme au Québec, par une mobilisation forte de leur environnement (Perron \& Veillette 2011; Bourdon \& Baril 2016). On ne trouve pas ici trace de tables de concertations territoriales mobilisant entreprises, tiers-secteur, et acteurs politiques locaux autour de la question du décrochage. Ce constat ne vaut évidemment que pour le terrain qui nourrit notre réflexion et qui s'ancre dans un espace régional, celui du Latium. C'est une limite de cette enquête qui mériterait un approfondissement comparatif interrégional.

La conception de la dispersion scolaire, ancrée dans une vision inclusive de l'école, confère-t-elle pour autant aux politiques publiques et aux stratégies d'établissements une texture particulière ? La réponse ici n'est pas aisée. Notons d'abord que l'on constate une grande régularité des instruments mobilisés et donc une faible dimension innovatrice liée au pilotage par appel d'offre. Les exercices de planification imposés aux établissements (Plan de l'offre triennale de formation, plan d'amélioration, plans annuels d'inclusion notamment) ouvrent un espace réflexif pour construire cette adéquation entre référentiel inclusif et dispositifs de soutien à la persévérance scolaire. Cette adéquation apparaît clairement à la lecture de ces documents. Par ailleurs, les dispositifs de type portail d'aide ou encore les actions de formation généralisées par la loi Buona Scuola s'inscrivent pleinement dans ce référentiel inclusif. Cependant, les pratiques jouant sur le temps scolaire ou les capacités d'encadrement ne se démarquent pas par leur ancrage dans un tel référentiel. En définitive, pour conclure de manière plus robuste sur ce point, il faudrait conduire un travail d'observation ethnographique dans les classes, qui n'a pas été possible dans le cadre de cette recherche exploratoire. Cela en constitue clairement une autre limite.

Pour finir, les grands absents de cette étude sont évidemment les élèves italiens et leurs familles. Les intégrer pleinement à une recherche sur le décrochage suppose de construire une relation de confiance impossible dans le cadre d'une enquête conduite dans une langue qui demeure, quoi qu'on en dise, étrangère quand elle n'est pas maternelle. Ils sont pourtant les premiers concernés et ils sont très rarement associés à la production des ressources 
qui leur sont proposées, ce qui explique sans doute qu'ils renâclent parfois à y recourir.

\section{Bibliographie}

Albanese O. \& Pieri M. (2013), «Les enseignants de soutien en Italie: leur formation aux methodes et philosophies inclusives », Canadian Journal of Education 36 (1) pp. 349- 74.

Bourdon S. \& Baril D. (2016), L'intervention des acteurs non scolaires dans le soutien à la persévérance scolaire. Synthèse des connaissances, Rapport remis au Secrétariat à la jeunesse du Québec, OJS-CERTA.

Boussaguet L., Jacquot S. \& Ravinet P. (Dir.) (2006), Dictionnaire des politiques publiques, Paris, Presses de Sciences Po, $2^{\text {ème }}$ édition.

Camera dei Deputati (2014), Doc. XVII, n 6, Documento approvato dalla VII commissione permanente (cultura, scienza e istruzione) Nella seduta del 21 ottobre 2014 a conclusione dell'indagine conoscitiva Deliberata nella seduta del 16 aprile 2014 Sulle strategie per contrastare la dispersione scolastica (articolo 144, comma 3, del regolamento della camera dei deputati)

Cobb R. W, Elder C. D. (1975), Participation in American Politics. The Dynamics of Agenda Building, Baltimore, The Johns Hopkins University Press, Baltimore.

Colombo M., (2013), "Disadvantaged Life Itineraries and the Use of Personal Agency Among Italian Early School Leavers and At-risk Students", Estudios Sobre Education, 24, pp. 9-35.

De Anna L. (2009), «La situation de l'inclusion scolaire en Italie : qu'est-ce qui fait encore débat?», La Nouvelle revue de l'adaptation et de la scolarisation, pp. 185- 91.

De Feudis E. (2017), Les politiques régionales de lutte contre le décrochage scolaire en Italie, France et Espagne, Thèse de science politique, Université la Cattolica de Milan.

Fondazione Giacomo Brodolini (2007), Interventi contro le ripetenze e la dispersione scolastica. l progetto r.i.di.sco nella provincia di Roma, A cura di Paola Mengoli, Quaderni della Fondazione G. Brodolini. 
Ghezzi S. et E. Mingione (2007), "Embeddedness, path dependancy and social institutions. An economic sociology approach", Current Sociology 55, pp. 11- 23.

INDIRE (2016), Crescere in coesione. Promuovere il successo scolastico, le pari opportunità e l'inclusione sociale: Sviluppo di reti contro la dispersione scolastica e creazione di prototipi innovativi, A cura di Patrizia Lotta \& Valentina Pedani, Rome, http://datipon.indire.it/cruscotti_f3/files/F3_rapporto\%20di\%20monitoraggi o\%20e\%20analisi\%20dei\%20prototipi_27_05_2016.pdf.

ISFOL (2012), Le azioni del PON 'competenze per lo sviluppo' di contrasto alla dispersione scolastica. Un'indagine valutativa, ISFOL, Janvier. http://isfoloa.isfol.it/handle/123456789/134?show=full

LOST (2014), Dispersione scolastica: il costo per la collettività e il ruolo di scuole e Terzo settore, A cura di Daniele Checchi, Milan,

Jobert B. \& Muller P. (1987), L'État en action, corporatismes et politiques publiques, Paris, Presses Universitaires de France.

MIUR (2013), Focus 'La dispersione scolastica', Rome, Juin. https://www.minori.it/sites/default/files/dati_miur_dispersione_scolastica.pd f

MIUR (2017a), Gli alunni stranieri nel sistema scolastico italiano a.s. 2015/2016, Rome, Mars. https://www.miur.gov.it/-/gli-alunni-stranieri-nelsistema-scolastico-italiano-a-s-2015-2016

MIUR (2017b), "Per la Scuola, competenze e ambienti per l'apprendimento", Programmazione 2014 - 2020 FSE - Inclusione sociale e lotta al disagio. Dati in sintesi, Rome, Juillet. http://89.97.132.197/fondistrutturali/secure/allegati/cds_2018/08_05_18_5.0 a_Rapporto_Monitoraggio.pdf

MIUR - INDIRE (2014), La lotta all'abbandono precoce dei percorsi di istruzione e formazione in Europa. Strategie, politiche e misure, Quaderni di Eurydice. http://eurydice.indire.it/pubblicazioni/la-lotta-allabbandonoprecoce-dei-percorsi-di-istruzione-e-formazione-in-europa-strategie-

politiche-e-misure-2016/

Muller P. (2000), «L'analyse cognitive des politiques publiques : vers une sociologie politique de l'action publique », Revue Française de Science Politique, 50-2, pp. 189-207 
Neveu É. (2015), Sociologie politique des problèmes publics, Collection "U", Paris, Armand Colin.

Palier B. (2010), "Path dependence (Dépendance au chemin emprunté)", in Dictionnaire des politiques publiques, Paris, Presses de Sciences Po, 3e éd, pp. 411- 19.

Perron M. \& Veillette S. (2011), «Territorialité, mobilisation des acteurs et persévérance scolaire: le cas du Conseil régional de prévention de l'abandon scolaire au Saguenay-Lac-Saint- Jean », Économie et Solidarités, 411-2, pp. 104-127.

Sabatier P. \& Schlager E. (2000), «Les approches cognitives des politiques publiques: perspectives américaines », Revue Française de Science Politique, 50-2, pp. 209-23.

Save the children (2011), Progetto InContro. Insieme contro la dispersione scolastica, Analisi e strategie di intervento per il contrasto alla dispersione scolastica, A cura di Katia Scannavini, Rome, Juin, https://s3.savethechildren.it/public/files/uploads/pubblicazioni/progettocontro-insieme-contro-la-dispersione-scolastica.pdf. 